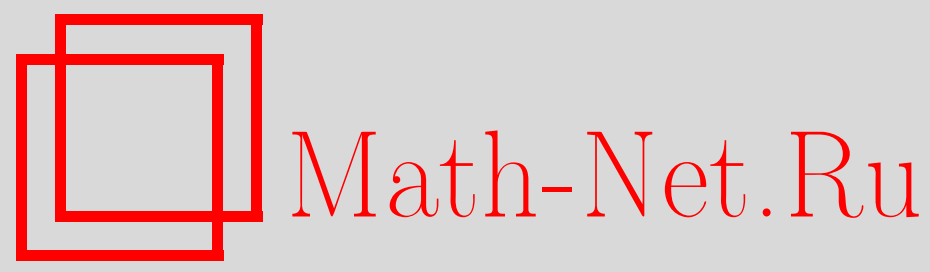

В. Ю. Попов, Неразрешимость проблемы равенства в относительно свободных кольцах, Матем. заметки, 2000, том 67, выпуск 4, 582-594

DOI: https://doi.org/10.4213/mzm873

Использование Общероссийского математического портала Math-Net.Ru подразумевает, что вы прочитали и согласны с пользовательским соглашением http://www.mathnet.ru/rus/agreement

Параметры загрузки:

IP : 34.239 .49 .27

26 апреля 2023 г., 09:43:36

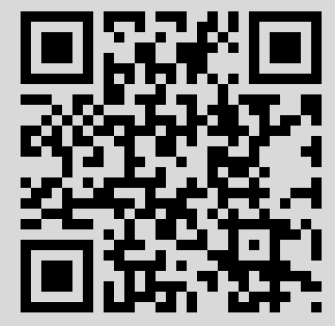




\title{
НЕРАЗРЕШИМОСТЬ ПРОБЛЕМЫ РАВЕНСТВА В ОТНОСИТЕЛЬНО СВОБОДНЫХ КОЛЬЦАХ
}

\author{
В. Ю. Попов
}

Построен пример серии конечно базируемых многообразий колец $\mathfrak{X}_{p}$ таких, что проблема равенства в кольце $F_{n} \mathfrak{X}_{p}$ ранга $n$, свободном в многообразии $\mathfrak{X}_{p}$, разрешима тогда и только тогда, когда $n<p$.

Библиограбфия: 2 названия.

В многообразиях колец с неразрешимой эквациональной теорией, построенных в работе [1], проблема равенства в свободном кольце ранга $p$ неразрешима для любого натурального $p$. Очевидно, что не существует многообразия колец такого, что $p>q$ и проблема равенства в свободном кольце ранга $p$ разрешима, а проблема равенства в свободном кольце ранга $q$ неразрешима. Теорема 1 дает пример серии конечно базируемых многообразий колец $\mathfrak{X}_{p}$ таких, что проблема равенства в кольце $F_{n} \mathfrak{X}_{p}$ ранга $n$, свободном в многообразии $\mathfrak{X}_{p}$, разрешима тогда и только тогда, когда $n<p$. В теореме 2 строится пример бесконечно базируемого многообразия колец с неразрешимой эквациональной теорией такого, что проблема равенства в свободном кольце ранга $p$ разрешима для любого натурального $p$. Заметим, что аналогичньй пример конечно базируемого многообразия колец автору неизвестен.

Теорема 1. Для любого натурального р существует конечно базируемое многообразие колеи $\mathfrak{X}_{p}$ такое, что проблема равенства в кольче $F_{n} \mathfrak{X}_{p}$ ранга $n$, свободном в многообразии $\mathfrak{X}_{p}$, разрешима тогда $u$ только тогда, когда $n<p$.

ДокАЗАТЕЛЬСТво. Пусть

$$
\begin{gathered}
\left.s_{1}\left(x_{1}, \ldots, x_{k}\right)=\left(\left(\left(\left(\ldots\left(\left(x_{1} x_{2}\right) x_{3}\right)\left(x_{4} x_{5}\right)\right) x_{6} \ldots\right) x_{k-5}\right)\left(\left(x_{k-4} x_{k-3}\right)\left(x_{k-2} x_{k-1}\right)\right)\right) x_{k}\right), \\
s_{2}\left(x_{1}, \ldots, x_{k+30}\right)=s_{1}\left(s_{1}\left(x_{1}, \ldots, x_{30}\right), x_{31}, \ldots, x_{k+30}\right), \\
s_{3}\left(x_{1}, \ldots, x_{p}\right)=x_{p}\left(\left(x_{p-2}\left(\ldots\left(x_{5}\left(\left(x_{3}\left(x_{1} x_{2}\right)\right) x_{4}\right)\right) x_{6} \ldots\right)\right) x_{p-1}\right), \\
t_{k}^{p}\left(x_{1}, \ldots, x_{p+k+60}\right)=s_{2}\left(s_{3}\left(s_{1}\left(x_{1}, \ldots, x_{30}\right), x_{31}, \ldots, x_{p+30}\right), x_{p+31}, \ldots, x_{p+k+60}\right) .
\end{gathered}
$$

Если $x_{i}=x$ при $1 \leqslant i \leqslant 33$ и $p+29 \leqslant i \leqslant p+k+60$, то $t_{k}^{p}\left(x_{1}, \ldots, x_{p+k+60)}\right.$ будем 
обозначать через $t_{k}^{p}\left(x_{34}, \ldots, x_{p+28} \mid x\right)$. Пусть

$$
\begin{aligned}
& A\left(x_{1}, \ldots, x_{p-6}, y\right)=A(\vec{x}, y)=t_{20}^{p}\left(x_{34}, \ldots, x_{p-6} \mid y\right), \\
& B\left(x_{1}, \ldots, x_{p-6}, y\right)=B(\vec{x}, y)=t_{24}^{p}\left(x_{34}, \ldots, x_{p-6} \mid y\right), \\
& C\left(x_{1}, \ldots, x_{p-6}, y\right)=C(\vec{x}, y)=t_{28}^{p}\left(x_{34}, \ldots, x_{p-6} \mid y\right), \\
& D\left(x_{1}, \ldots, x_{p-6}, y\right)=D(\vec{x}, y)=t_{32}^{p}\left(x_{34}, \ldots, x_{p-6} \mid y\right), \\
& Q_{i}\left(x_{1}, \ldots, x_{p-6}, y\right)=Q_{i}(\vec{x}, y)=t_{36+4 i}^{p}\left(x_{34}, \ldots, x_{p-6} \mid y\right) ; \\
& W=\left\{A(\vec{x}, y), B(\vec{x}, y), C(\vec{x}, y), D(\vec{x}, y), Q_{i}(\vec{x}, y) \mid i \in\{0,1, \ldots, m\}\right\} .
\end{aligned}
$$

Если не оговорено противное, то будем считать, что

$$
w_{1} \ldots w_{n}=\left(\left(\ldots\left(\left(w_{1} w_{2}\right) w_{3}\right) \ldots\right) w_{n}\right), \quad w_{1} w_{2}^{n}=\left(\left(\ldots\left(\left(w_{1} w_{2}\right) w_{2}\right) \ldots\right) w_{2}\right)
$$

Зафиксируем некоторое рекурсивно перечислимое нерекурсивное множество натуральных чисел $P$. Через $M$ обозначим двухленточную машину Минского, вычисляющую частичную характеристическую функцию множества $P$. Будем считать, что число внутренних состояний машины $M$ равно $m+1$.

Произвольной команде $q_{i} \delta_{1} \delta_{2} \rightarrow q_{j} \varepsilon_{1} \varepsilon_{2}$ машины $M$ поставим в соответствие некоторое кольцевое тождество:

$Q_{i}(\vec{x}, y) C(\vec{x}, y) D(\vec{x}, y)=Q_{j}(\vec{x}, y) A^{\varepsilon_{1}}(\vec{x}, y) B^{\varepsilon_{2}}(\vec{x}, y) C(\vec{x}, y) D(\vec{x}, y), \quad$ если $\delta_{1}=1, \delta_{2}=1$, $Q_{i}(\vec{x}, y) A(\vec{x}, y) D(\vec{x}, y)=Q_{j}(\vec{x}, y) A^{\varepsilon_{1}+1}(\vec{x}, y) B^{\varepsilon_{2}}(\vec{x}, y) D(\vec{x}, y), \quad$ если $\delta_{1}=1, \delta_{2}=0$, $Q_{i}(\vec{x}, y) B(\vec{x}, y) C(\vec{x}, y)=Q_{j}(\vec{x}, y) A^{\varepsilon_{1}}(\vec{x}, y) B^{\varepsilon_{2}+1}(\vec{x}, y) C(\vec{x}, y), \quad$ если $\delta_{1}=0, \delta_{2}=1$, $Q_{i}(\vec{x}, y) A(\vec{x}, y) B(\vec{x}, y)=Q_{j}(\vec{x}, y) A^{\varepsilon_{1}+1}(\vec{x}, y) B^{\varepsilon_{2}+1}(\vec{x}, y), \quad$ если $\delta_{1}=0, \delta_{2}=0$.

Обозначим через $\mathfrak{M}^{p}$ многообразие колец, заданное тождествами, кодирующими команды машины $M$; через $\mathfrak{B}^{p}$ - многообразие колец, заданное тождествами:

$$
\begin{aligned}
& z A(\vec{x}, y) B(\vec{x}, y)=z B(\vec{x}, y) A(\vec{x}, y), \quad z C(\vec{x}, y) D(\vec{x}, y)=z D(\vec{x}, y) C(\vec{x}, y) \\
& z A(\vec{x}, y) D(\vec{x}, y)=z D(\vec{x}, y) A(\vec{x}, y), \quad z B(\vec{x}, y) C(\vec{x}, y)=z C(\vec{x}, y) B(\vec{x}, y)
\end{aligned}
$$

через $\mathfrak{R}_{2}$ - многообразие всех колец характеристики 2 . Пусть, наконец, $\mathfrak{D}^{p}$ - многообразие, заданное тождествами: $t_{k}^{p}\left(x_{1}, \ldots, x_{p+k+60}\right)=0$, если $x_{i}=x_{j}$ при $34 \leqslant i \leqslant j \leqslant p+28$ или $x_{i}=y_{1} y_{2}$ при $34 \leqslant i \leqslant p+28$, где $k \in\{20+4 i \mid i \in\{0,1, \ldots, m+4\}\}$. Обозначим через $\mathfrak{X}_{p}$ многообразие $\mathfrak{M}^{p+6} \cap \mathfrak{B}^{p+6} \cap \mathfrak{D}^{p+6} \cap \mathfrak{R}_{2}$.

Лемма 1. Если $n<p$, то проблема равенства в кольие $F_{n} \mathfrak{X}_{p}$ разрешима.

ДокАЗАТЕЛЬСТво. Пусть $F_{n} \mathfrak{D}^{k} \cap \mathfrak{R}_{2}-$ кольцо ранга $n$, свободное в многообразии $\mathfrak{D}^{k} \cap \mathfrak{R}_{2}$. Покажем, что кольцо $F_{n} \mathfrak{X}_{p}$ изоморфно кольцу $F_{n} \mathfrak{D}^{p+6} \cap \mathfrak{R}_{2}$. Для этого, очевидно, достаточно показать, что $F_{n} \mathfrak{D}^{p+6} \cap \mathfrak{R}_{2}$ принадлежит многообразию $\mathfrak{X}_{p}$. Пусть $f(\vec{x})=0$ - одно из тождеств, задающих многообразие $\mathfrak{M}^{p+6} \cap \mathfrak{B}^{p+6}$. Тогда каждый одночлен многочлена $f(\vec{x})$ содержит подслово вида $t_{k}^{p+6}\left(x_{1}, \ldots, x_{(p+6)+k+60}\right)$. Покажем, что для любых $x_{1}, \ldots, x_{p+k+66}$ в кольце $F_{n} \mathfrak{D}^{p+6} \cap \mathfrak{R}_{2}$ вьполняется соотношение 
$t_{k}^{p+6}\left(x_{1}, \ldots, x_{(p+6)+k+60}\right)=0$. Представим многочлен $t_{k}^{p+6}\left(x_{1}, \ldots, x_{(p+6)+k+60}\right)$ в виде суммы одночленов $\sum_{i} t_{k}^{p+6}\left(u_{1}^{i}, \ldots, u_{(p+6)+k+60}^{i}\right)$. Рассмотрим слова $u_{j}^{i}$ при $34 \leqslant j \leqslant$ $p+34$ и произвольном, но фиксированном $i$. Из тождеств многообразия $\mathfrak{D}^{p+6}$ очевидным образом вытекает, что $t_{k}^{p+6}\left(u_{1}^{i}, \ldots, u_{(p+6)+k+60}^{i}\right)=0$, если $u_{j_{1}}^{i}=u_{j_{2}}^{i}$ при $j_{1} \neq j_{2}$ или если $u_{j}^{i}=v_{1} v_{2}$ для некоторых слов $v_{1}$ и $v_{2}$. Поэтому либо для любого $j_{1}, j_{2} \in\{34, \ldots, p+34\} u_{j_{1}}^{i} \neq u_{j_{2}}^{i}-$ свободные образуюшие кольца $F_{n} \mathfrak{D}^{p+6} \cap \mathfrak{R}_{2}$, либо $t_{k}^{p+6}\left(u_{1}^{i}, \ldots, u^{i}(p+6)+k+60\right)=0$. По условию леммы $n<p$. Следовательно, кольцо $F_{n} \mathfrak{D}^{p+6} \cap \mathfrak{R}_{2}$ не имеет $p$ попарно различных свободных образующих, а значит, $t_{k}^{p+6}\left(u_{1}^{i}\right.$, $\ldots, u_{(p+6)+k+60}^{i}=0$. В силу произвольности $i$ отсюда вытекает, что

$$
t_{k}^{p+6}\left(x_{1}, \ldots, x_{(p+6)+k+60}\right)=\sum_{i} t_{k}^{p+6}\left(u_{1}^{i}, \ldots, u_{(p+6)+k+60}^{i}\right)=0 .
$$

Таким образом, тождество $f(\vec{x})=0$ действительно вьполняется в кольце $F_{n} \mathfrak{D}^{p+6} \cap \mathfrak{R}_{2}$. Итак, мы показали, что кольца $F_{n} \mathfrak{X}_{p}$ и $F_{n} \mathfrak{D}^{p+6} \cap \mathfrak{R}_{2}$ изоморфны. Следовательно, нам достаточно убедиться в разрешимости проблемы равенства в кольце $F_{n} \mathfrak{D}^{p+6} \cap \Re_{2}$.

Пусть $S$ - множество многочленов $f(\vec{e})$, полученных подстановкой конкретных элементов кольца $F_{n} \mathfrak{D}^{p+6} \cap \mathfrak{R}_{2}$ в тождества, определяющие многообразие $\mathfrak{D}^{p+6}$, где $\left\{e_{1}\right.$, $\left.\ldots, e_{n}\right\}$ - множество свободных образуюших кольца $F_{n} \mathfrak{D}^{p+6} \cap \mathfrak{R}_{2}$. Обозначим через $I$ идеал кольца $F_{n} \Re_{2}$ ранга $n$, свободного в многообразии $\mathfrak{R}_{2}$, порожденньй множеством $S$. Соотношение $g(\vec{e})=0$ выполняется в кольце $F_{n} \mathfrak{D}^{p+6} \cap \mathfrak{R}_{2}$ тогда и только тогда, когда многочлен $g(\vec{e})$ принадлежит идеалу $I$. Соотношение $F_{n} \Re_{2}=g(\vec{e}) \in I$ равносильно вьполнению в кольце $F_{n} \Re_{2}$ равенства вида $g(\vec{e})=\sum_{i, j} u_{i_{1}} h_{j} u_{i_{2}}$, где $h_{j} \in S$, $u_{i_{1}} h_{j} u_{i_{2}}$ - некоторое произведение конечного числа многочленов, одним из сомножителей которого является многочлен $h_{j}$. В силу дистрибутивности можно считать, что все многочлены, входяшие в произведение $u_{i_{1}} h_{j} u_{i_{2}}$, кроме многочлена $h_{j}$, являются одночленами. Заметим, что так как многочлен $h_{j}$ принадлежит множеству $S$, то каждьй одночлен многочлена $h_{j}$ содержит подслово вида $t_{k}^{p+6}\left(x_{1}, \ldots, x_{(p+6)+k+60}\right)$. Отсюданепосредственно вытекает, что каждьй одночлен многочлена $\sum_{i, j} u_{i_{1}} h_{j} u_{i_{2}}$ содержит подслово вида $t_{k}^{p+6}\left(x_{1}, \ldots, x_{(p+6)+k+60}\right)$. Следовательно, для того чтобы вьполнялось равенство $g(\vec{e})=\sum_{i, j} u_{i_{1}} h_{j} u_{i_{2}}$, необходимо, чтобы каждьй одночлен многочлена $g(\vec{e})$ содержал подслово вида $t_{k}^{p+6}\left(x_{1}, \ldots, x_{(p+6)+k+60}\right)$. Так как проблема равенства в кольце $F_{n} \Re_{2}$ разрешима, то сушествует алгоритм, определяющий по произвольному многочлену $g(\vec{e})$ содержат ли его одночлены подслова вида $t_{k}^{p+6}\left(x_{1}, \ldots, x_{(p+6)+k+60}\right)$. Итак, если какой-либо одночлен многочлена $g(\vec{e})$ не содержит подслова вида $t_{k}^{p+6}\left(x_{1}, \ldots\right.$, $\left.x_{(p+6)+k+60}\right)$, то в кольце $F_{n} \Re_{2}$ не вьполняется равенство $g(\vec{e})=\sum_{i, j} u_{i_{1}} h_{j} u_{i_{2}}$ ни для какого многочлена $\sum_{i, j} u_{i_{1}} h_{j} u_{i_{2}}$, а значит, в кольце $F_{n} \mathfrak{D}^{p+6} \cap \mathfrak{R}_{2}$ не вьполняется равенство $g(\vec{e})=0$. Пусть теперь каждьй одночлен многочлена $g(\vec{e})$ содержит подслово вида $t_{k}^{p+6}\left(x_{1}, \ldots, x_{(p+6)+k+60}\right)$. Индукцией по числу $d$ одночленов многочлена $g(\vec{e})$ покажем, что для многочлена $g(\vec{e})$ сушествует многочлен $\sum_{i, j} u_{i_{1}} h_{j} u_{i_{2}}$ такой, что $g(\vec{e})=\sum_{i, j} u_{i_{1}} h_{j} u_{i_{2}}$ - истинное равенство в кольце $F_{n} \Re_{2}$. Пусть $d=1$. Рассмотрим подслова $x_{i}$ при $34 \leqslant i \leqslant p+34$ слова $t_{k}^{p+6}\left(x_{1}, \ldots, x_{(p+6)+k+60}\right)$, являющегося подсловом одночлена многочлена $g(\vec{e})$. Допустим, что существуют $i$ и $l$ такие, что $34 \leqslant i<l \leqslant p+34$ и $x_{i}=x_{l}$. Тогда по построению многообразия $\mathfrak{D}^{p+6}$ слово $t_{k}^{p+6}\left(x_{1}, \ldots, x_{(p+6)+k+60}\right)$ принадлежит множеству $S$, и, следовательно, многочлен 
$\sum_{i, j} u_{i_{1}} h_{j} u_{i_{2}}$ существует. Предположим теперь, что для любых $i, l$ таких, что $34 \leqslant i<$ $l \leqslant p+34, x_{i} \neq x_{l}$. Так как $n<p$, то сушествует $i$ такое, что $x_{i}$ не является свободным образующим кольца $F_{n} \mathfrak{R} 2$. Поэтому $x_{i}$ можно представить в виде $y_{i_{1}} y_{i_{2}}$. Отсюда вытекает, что $t_{k}^{p+6}\left(x_{1}, \ldots, x_{(p+6)+k+60}\right)$ принадлежит множеству $S$, а значит, многочлен $\sum_{i, j} u_{i_{1}} h_{j} u_{i_{2}}$ существует. База индукции доказана. Допустим, что утверждение справедливо для всех $d$ меньших $d_{0}$. Покажем, что оно выполняется и для $d_{0}$. В самом деле, пусть многочлен $g(\vec{e})$ имеет ровно $d_{0}$ одночленов. Тогда многочлен $g(\vec{e})$ представим в виде суммы двух многочленов $g_{1}(\vec{e})+g_{2}(\vec{e})$, где число одночленов многочлена $g_{i}(\vec{e})$ равно $d_{i}, d_{1}+d_{2}=d_{0}, d_{i} \neq 0$. Так как $d_{0}>d_{i}$, то для многочленов $g_{1}(\vec{e})$ и $g_{2}(\vec{e})$ справедливо предположение индукции. Следовательно, $g_{1}(\vec{e})=\sum_{i, j} u_{i_{1}} h_{j} u_{i_{2}}, g_{2}(\vec{e})=\sum_{i, j} v_{i_{1}} h_{j} v_{i_{2}}$ и $\sum_{i, j} u_{i_{1}} h_{j} u_{i_{2}}+\sum_{i, j} v_{i_{1}} h_{j} v_{i_{2}}$ - требуемый многочлен для $g(\vec{e})$.

Таким образом, $F_{n} \mathfrak{D}^{p+6} \cap \mathfrak{R}_{2}=g(\vec{e})=0$ тогда и только тогда, когда каждый одночлен многочлена $g(\vec{e})$ содержит подслово вида $t_{k}^{p+6}\left(x_{1}, \ldots, x_{(p+6)+k+60}\right)$. Следовательно, проблема равенства в кольце $F_{n} \mathfrak{D}^{p+6} \cap \mathfrak{R}_{2}$ разрешима. Лемма 1 доказана.

Лемма 2. Если машина $M$ через конечное число тактов работы переходит из конфигурачии $q_{i} \xi_{1} \xi_{2}$ в конфигурацию $q_{j} \eta_{1} \eta_{2}$, то в многообразии $\mathfrak{X}_{p}$ выполняется тохдество

$$
Q_{i}(\vec{x}, y) A^{\xi_{1}}(\vec{x}, y) B^{\xi_{2}}(\vec{x}, y) C(\vec{x}, y) D(\vec{x}, y)=Q_{j}(\vec{x}, y) A^{\eta_{1}}(\vec{x}, y) B^{\eta_{2}}(\vec{x}, y) C(\vec{x}, y) D(\vec{x}, y)
$$

ДокАЗАТЕЛЬСТво. Доказательство проведем индукцией по числу $r$ тактов работы машины $M$. Пусть $r=0$. Тогда утверждение очевидно. Предположим, что утверждение леммы справедливо для некоторого $r$. Покажем, что оно вьполняется и для $r+1$. Пусть через $r$ тактов машина $M$ переходит из конфигурации $q_{i} \xi_{1} \xi_{2}$ в конфигурацию $q_{l} \tau_{1} \tau_{2}$ и на $(r+1)$-м шаге вьполняется команда $q_{l} \delta_{1} \delta_{2} \rightarrow q_{j} \varepsilon_{1} \varepsilon_{2}$. Следует рассмотреть четыре случая в зависимости от значений $\delta_{1}$ и $\delta_{2}$. Допустим, что $\delta_{1}=1$ и $\delta_{2}=0$. Остальные случаи рассматриваются аналогично. Тогда по определению машины $M$ $\tau_{1}=0, \tau_{2}>0$, и в многообразии $\mathfrak{X}_{p}$ вьполняется тождество

$$
Q_{i}(\vec{x}, y) A^{\xi_{1}}(\vec{x}, y) B^{\xi_{2}}(\vec{x}, y) C(\vec{x}, y) D(\vec{x}, y)=Q_{l}(\vec{x}, y) A^{\tau_{1}}(\vec{x}, y) B^{\tau_{2}}(\vec{x}, y) C(\vec{x}, y) D(\vec{x}, y)
$$

Отсюда получаем

$$
\begin{aligned}
Q_{i}( & \vec{x}, y) A^{\xi_{1}}(\vec{x}, y) B^{\xi_{2}}(\vec{x}, y) C(\vec{x}, y) D(\vec{x}, y) \\
& =Q_{l}(\vec{x}, y) B^{\tau_{2}}(\vec{x}, y) C(\vec{x}, y) D(\vec{x}, y)=Q_{l}(\vec{x}, y) B(\vec{x}, y) C(\vec{x}, y) B^{\tau_{2}-1}(\vec{x}, y) D(\vec{x}, y) \\
& =Q_{j}(\vec{x}, y) A^{\varepsilon_{1}}(\vec{x}, y) B^{\varepsilon_{2}+1}(\vec{x}, y) C(\vec{x}, y) B^{\tau_{2}-1}(\vec{x}, y) D(\vec{x}, y) \\
& =Q_{j}(\vec{x}, y) A^{\varepsilon_{1}}(\vec{x}, y) B^{\varepsilon_{2}+\tau_{2}}(\vec{x}, y) C(\vec{x}, y) D(\vec{x}, y) \\
& =Q_{j}(\vec{x}, y) A^{\eta_{1}}(\vec{x}, y) B^{\eta_{2}}(\vec{x}, y) C(\vec{x}, y) D(\vec{x}, y) .
\end{aligned}
$$

В данной цепочке равенств первое равенство выполняется в силу тождества

$$
Q_{i}(\vec{x}, y) A^{\xi_{1}}(\vec{x}, y) B^{\xi_{2}}(\vec{x}, y) C(\vec{x}, y) D(\vec{x}, y)=Q_{l}(\vec{x}, y) A^{\tau_{1}}(\vec{x}, y) B^{\tau_{2}}(\vec{x}, y) C(\vec{x}, y) D(\vec{x}, y)
$$


и равенства $\tau_{1}=0$, второе и четвертое - в силу тождества

$$
z B(\vec{x}, y) C(\vec{x}, y)=z C(\vec{x}, y) B(\vec{x}, y)
$$

третье - в силу тождества

$$
Q_{l}(\vec{x}, y) B(\vec{x}, y) C(\vec{x}, y)=Q_{j}(\vec{x}, y) A^{\varepsilon_{1}}(\vec{x}, y) B^{\varepsilon_{2}+1}(\vec{x}, y) C(\vec{x}, y)
$$

пятое - поскольку $\tau_{1}=0$ и по определению машины $M \eta_{i}=\tau_{i}+\varepsilon_{i}$. Лемма 2 доказана.

Предположим, что многообразие $\mathfrak{X}_{p}$ удовлетворяет тождеству

$$
Q_{i}(\vec{x}, y) A^{\xi_{1}}(\vec{x}, y) B^{\xi_{2}}(\vec{x}, y) C(\vec{x}, y) D(\vec{x}, y)=Q_{j}(\vec{x}, y) A^{\eta_{1}}(\vec{x}, y) B^{\eta_{2}}(\vec{x}, y) C(\vec{x}, y) D(\vec{x}, y)
$$

Тогда и кольцо счетного ранга $F \mathfrak{X}_{p}$, свободноев многообразии $\mathfrak{X}_{p}$, удовлетворяет этому тождеству, а значит, и соотношению

$$
\begin{aligned}
& Q_{i}\left(\vec{e}, e_{1}\right) A^{\xi_{1}}\left(\vec{e}, e_{1}\right) B^{\xi_{2}}\left(\vec{e}, e_{1}\right) C\left(\vec{e}, e_{1}\right) D\left(\vec{e}, e_{1}\right) \\
& \quad=Q_{j}\left(\vec{e}, e_{1}\right) A^{\eta_{1}}\left(\vec{e}, e_{1}\right) B^{\eta_{2}}\left(\vec{e}, e_{1}\right) C\left(\vec{e}, e_{1}\right) D\left(\vec{e}, e_{1}\right)
\end{aligned}
$$

где $\vec{e}=\left(e_{1}, \ldots, e_{s}\right)$.

Обозначим через $S$ множество многочленов $f(\vec{e})$ таких, что $f(\vec{e})=0$-соотношение в кольце $F \mathfrak{X}_{p}$, полученное подстановкой конкретных значений вместо переменных в одно из тождеств, задающих многообразие $\mathfrak{M}^{p+6} \cap \mathfrak{B}^{p+6} \cap \mathfrak{D}^{p+6}$. Пусть $I$ - идеал, порожденньй множеством $S$ в кольце $F \Re_{2}$. Тогда очевидно, что

$$
\begin{aligned}
F \mathfrak{X}_{p}=Q_{i}\left(\vec{e}, e_{1}\right) A^{\xi_{1}}\left(\vec{e}, e_{1}\right) B^{\xi_{2}}\left(\vec{e}, e_{1}\right) C\left(\vec{e}, e_{1}\right) D\left(\vec{e}, e_{1}\right) \\
=Q_{j}\left(\vec{e}, e_{1}\right) A^{\eta_{1}}\left(\vec{e}, e_{1}\right) B^{\eta_{2}}\left(\vec{e}, e_{1}\right) C\left(\vec{e}, e_{1}\right) D\left(\vec{e}, e_{1}\right) \\
\Longleftrightarrow \quad F \Re_{2}=Q_{i}\left(\vec{e}, e_{1}\right) A^{\xi_{1}}\left(\vec{e}, e_{1}\right) B^{\xi_{2}}\left(\vec{e}, e_{1}\right) C\left(\vec{e}, e_{1}\right) D\left(\vec{e}, e_{1}\right) \\
\quad+Q_{j}\left(\vec{e}, e_{1}\right) A^{\eta_{1}}\left(\vec{e}, e_{1}\right) B^{\eta_{2}}\left(\vec{e}, e_{1}\right) C\left(\vec{e}, e_{1}\right) D\left(\vec{e}, e_{1}\right) \in I .
\end{aligned}
$$

Вхождение многочлена

$$
\begin{aligned}
& Q_{i}\left(\vec{e}, e_{1}\right) A^{\xi_{1}}\left(\vec{e}, e_{1}\right) B^{\xi_{2}}\left(\vec{e}, e_{1}\right) C\left(\vec{e}, e_{1}\right) D\left(\vec{e}, e_{1}\right) \\
& \quad+Q_{j}\left(\vec{e}, e_{1}\right) A^{\eta_{1}}\left(\vec{e}, e_{1}\right) B^{\eta_{2}}\left(\vec{e}, e_{1}\right) C\left(\vec{e}, e_{1}\right) D\left(\vec{e}, e_{1}\right)
\end{aligned}
$$

в идеал $I$ равносильно в кольце $F \Re_{2}$ равенству вида

$$
\begin{aligned}
& Q_{i}\left(\vec{e}, e_{1}\right) A^{\xi_{1}}\left(\vec{e}, e_{1}\right) B^{\xi_{2}}\left(\vec{e}, e_{1}\right) C\left(\vec{e}, e_{1}\right) D\left(\vec{e}, e_{1}\right) \\
& \quad+Q_{j}\left(\vec{e}, e_{1}\right) A^{\eta_{1}}\left(\vec{e}, e_{1}\right) B^{\eta_{2}}\left(\vec{e}, e_{1}\right) C\left(\vec{e}, e_{1}\right) D\left(\vec{e}, e_{1}\right)=\sum_{i, j} u_{i_{1}} h_{j} u_{i_{2}}
\end{aligned}
$$

где $u_{i_{1}} h_{j} u_{i_{2}}$ - некоторое произведение многочленов, одним из сомножителей которого является многочлен $h_{j}, h_{j} \in S$. 
Индукцией по числу $d$ одночленов в сумме $\sum_{i, j} u_{i_{1}} h_{j} u_{i_{2}}$ убедимся в том, что вьполняется равенство $\sum_{i, j} u_{i_{1}} h_{j} u_{i_{2}}=f_{1}+f_{2}$, где $f_{1}$-многочлен, каждьй одночлен которого может быть получен конечным числом перестановок вида

$$
\begin{aligned}
& A\left(\vec{e}, e_{1}\right) B\left(\vec{e}, e_{1}\right) \leftrightarrow B\left(\vec{e}, e_{1}\right) A\left(\vec{e}, e_{1}\right), \quad C\left(\vec{e}, e_{1}\right) D\left(\vec{e}, e_{1}\right) \leftrightarrow D\left(\vec{e}, e_{1}\right) C\left(\vec{e}, e_{1}\right), \\
& A\left(\vec{e}, e_{1}\right) D\left(\vec{e}, e_{1}\right) \leftrightarrow D\left(\vec{e}, e_{1}\right) A\left(\vec{e}, e_{1}\right), \quad B\left(\vec{e}, e_{1}\right) C\left(\vec{e}, e_{1}\right) \leftrightarrow C\left(\vec{e}, e_{1}\right) B\left(\vec{e}, e_{1}\right)
\end{aligned}
$$

из одночлена вида $Q_{l}\left(\vec{e}, e_{1}\right) A^{r_{1}}\left(\vec{e}, e_{1}\right) B^{r_{2}}\left(\vec{e}, e_{1}\right) C\left(\vec{e}, e_{1}\right) D\left(\vec{e}, e_{1}\right), f_{1}=\sum_{i, j} v_{i_{1}} h_{j} v_{i_{2}}, f_{2}-$ многочлен, ни один одночлен которого не удовлетворяет условиям, наложенным на одночлены многочлена $f_{1}$. Пусть $d=0$. Тогда утверждение очевидно. Допустим, что утверждение справедливо для некоторого $d$. Покажем, что оно верно и для $d+1$. Пусть в сумме $\sum_{i, j} u_{i_{1}} h_{j} u_{i_{2}}$ не более $d+1$ одночленов. Очевидно, что число одночленов в сумме $\sum_{i>1, j} u_{i_{1}} h_{j} u_{i_{2}}$ не больше $d$. Следовательно, по предположению индукции сумма $\sum_{i>1, j} u_{i_{1}} h_{j} u_{i_{2}}$ представима в виде $f_{1}+f_{2}$. Поэтому нам достаточно показать, что многочлен $u_{11} h_{1} u_{12}$ можно представить в виде $f_{1}+f_{2}$. В зависимости от того, получен ли многочлен $h_{1}$ из тождества многообразия $\mathfrak{M}^{p+6}, \mathfrak{B}^{p+6}$ или $\mathfrak{D}^{p+6}$, следует рассмотреть три случая.

В дальнейшем нам потребуется следуюшая

Лемма 3. Слово $w_{1}\left(\vec{e}, e_{1}\right) \ldots w_{n}\left(\vec{e}, e_{1}\right)$, әде $w_{i}\left(\vec{e}, e_{1}\right) \in W$, содержит подслово $t_{u}^{v}\left(x_{1}, \ldots, x_{u+v+60}\right)$ при $u \geqslant 20$ тогда и только тогда, когда $w_{i}\left(\vec{e}, e_{1}\right)=t_{u}^{v}\left(x_{1}, \ldots\right.$, $x_{u+v+60)}$ для некоторого $i \in\{1,2, \ldots, n\}$.

ДоказАтЕльство. Очевидно, что если существует $i$ такое, что $w_{i}\left(\vec{e}, e_{1}\right)=t_{u}^{v}\left(x_{1}, \ldots\right.$, $\left.x_{u+v+60}\right)$, то слово $t_{u}^{v}\left(x_{1}, \ldots, x_{u+v+60}\right)$ будет подсловом слова $w_{1}\left(\vec{e}, e_{1}\right) \ldots w_{n}\left(\vec{e}, e_{1}\right)$. Пусть теперь $t_{u}^{v}\left(x_{1}, \ldots, x_{u+v+60}\right)$ - подслово слова $w_{1}\left(\vec{e}, e_{1}\right) \ldots w_{n}\left(\vec{e}, e_{1}\right)$. Докажем индукцией по $n$, что $w_{i}\left(\vec{e}, e_{1}\right)=t_{u}^{v}\left(x_{1}, \ldots, x_{u+v+60}\right)$ для некоторого $i$. Пусть $n=1$. Тогда $t_{u}^{v}\left(x_{1}, \ldots, x_{u+v+60}\right)$ - подслово слова $w_{1}\left(\vec{e}, e_{1}\right)$. Заметим, что $t_{u}^{v}\left(x_{1}, \ldots, x_{u+v+60}\right)$ представимо в виде $\left(z_{1}\left(z_{2} z_{3}\right)\right) z_{4}$, где

$$
\begin{aligned}
z_{1}= & \left(\ldots\left(\left(s_{1}\left(s_{3}\left(s_{1}\left(x_{1}, \ldots, x_{30}\right), x_{31}, \ldots, x_{v+30}\right), x_{v+31}, \ldots, x_{v+60}\right) x_{v+61}\right) x_{v+62}\right)\right. \\
& \left.\left.\left(x_{v+63} x_{v+64}\right)\right) x_{v+65} \ldots\right) x_{u+v+55}, \\
z_{2}= & x_{u+v+56} x_{u+v+57}, \quad z_{3}=x_{u+v+58} x_{u+v+59}, \quad z_{4}=x_{u+v+60} .
\end{aligned}
$$

Следовательно, соответствующее подслово слова $w_{1}\left(\vec{e}, e_{1}\right)$ должно быть представимо в виде $\left(z_{1}\left(z_{2} z_{3}\right)\right) z_{4}$. Как нетрудно убедиться, подслов слова $w_{1}\left(\vec{e}, e_{1}\right)$, имеющих такой вид, возможно только девять:

1) $(((e e) e)(e e)) e$

2) $s_{1}(e, \ldots, e)$

3) $\left(e\left(s_{1}(e, \ldots, e) e\right)\right) e$;

4) $\left.\left.(\underbrace{e(\ldots(e}_{p-6 \text { ра3 }}\left(\left(e\left(s_{1}(e, \ldots, e) e\right)\right) e\right)) \ldots\right)\right) e$;

5) $s_{3}\left(s_{1}(e, \ldots, e), e, \ldots, e\right) e$

6) $\left(\left(\left(s_{3}\left(s_{1}(e, \ldots, e), e, \ldots, e\right) e\right) e\right)(e e)\right) e$;

7) $s_{1}\left(s_{3}\left(s_{1}(e, \ldots, e), \ldots, e\right), e, \ldots, e\right)$;

8) $\left(\left(\left(s_{1}\left(s_{3}\left(s_{1}(e, \ldots, e), \ldots, e\right), e, \ldots, e\right) e\right) e\right)(e e)\right) e$;

9) $w_{1}\left(\vec{e}, e_{1}\right)$, где $e \in\left\{e_{1}, e_{2}, \ldots\right\}$. 
Заметим, что длина слова $t_{u}^{v}\left(x_{1}, \ldots, x_{u+v+60}\right)$ больше 60, а длины слов 1$\left.), 2\right)$ и 3 ) равны 6,30 и 33, соответственно. Следовательно, случаи 1)-3) невозможны. Предположим, что имеет место случай 4). Тогда должны вьполняться следующие равенства:

$$
z_{1}=e, \quad z_{2}=e, \quad z_{3}=\underbrace{e(\ldots(e}_{p-8 \text { раз }}\left(\left(e\left(s_{1}(e, \ldots, e) e\right)\right) e\right)) \ldots), \quad z_{4}=e .
$$

Отсюда вытекает равенство $e=x_{u+v+56} x_{u+v+57}$, что невозможно. В случае 5) получаем

$$
z_{1}=e, \quad z_{2}=\underbrace{e(\ldots(e}_{p-6 \text { pa3 }}\left(\left(e\left(s_{1}(e, \ldots, e) e\right)\right) e\right)) \ldots), \quad z_{3}=e, \quad z_{4}=e .
$$

Отсюда следует ложное равенство $e=x_{u+v+58} x_{u+v+59}$. Если вьполняется случай 6$)$, то должны иметь место следующие равенства:

$$
z_{1}=\left(s_{3}\left(s_{1}(e, \ldots, e), e, \ldots, e\right) e\right) e, \quad z_{2}=e, \quad z_{3}=e, \quad z_{4}=e
$$

Следовательно, $e=x_{u+v+56} x_{u+v+57}$, что невозможно. Предположим, что имеет место случай 7). Тогда

$$
z_{1}=(\ldots\left(\left(\left(s_{3}\left(s_{1}(e, \ldots, e), e, \ldots, e\right) e\right) e\right)(e e)\right) \underbrace{e) \ldots) e}_{20 \text { раз }}, \quad z_{2}=e e, \quad z_{3}=e e, \quad z_{4}=4
$$

Отсюда вытекает, что

$$
\begin{aligned}
(\ldots & \left(\left(\left(s_{3}\left(s_{1}(e, \ldots, e), e, \ldots, e\right) e\right) e\right)(e e)\right) \underbrace{e) \ldots) e}_{20 \mathrm{pa}} \\
= & \left(\ldots \left(\left(\left(s_{1}\left(s_{3}\left(s_{1}\left(x_{1}, \ldots, x_{30}\right), x_{31}, \ldots, x_{v+30}\right), x_{v+31}, \ldots, x_{v+60}\right) x_{v+61}\right) x_{v+62}\right)\right.\right. \\
& \left.\left.\left(x_{v+63} x_{v+64}\right)\right) x_{v+65} \ldots\right) x_{u+v+55} .
\end{aligned}
$$

Легко понять, что последнее равенство имеет место тогда и только тогда, когда $u-9=20$, $x_{i}=e$ при $i \in\{v+61, \ldots, u+v+55\}$ и справедливо равенство

$$
s_{3}\left(s_{1}(e, \ldots, e), \ldots, e\right)=s_{1}\left(s_{3}\left(s_{1}\left(x_{1}, \ldots, x_{30}\right), x_{31}, \ldots, x_{v+30}\right), x_{v+31}, \ldots, x_{v+60}\right) .
$$

Из последнего равенства очевидньм образом вытекает

$$
\begin{aligned}
e= & \left(\left(\ldots\left(\left(s_{3}\left(s_{1}\left(x_{1}, \ldots, x_{30}\right), x_{31}, \ldots, x_{v+30}\right) x_{v+31}\right) x_{v+32}\right)\right.\right. \\
& \left.\left.\left.\left(x_{v+33} x_{v+34}\right)\right) x_{v+35} \ldots\right) x_{v+55}\right)\left(\left(x_{v+56} x_{v+57}\right)\left(x_{v+58} x_{v+59}\right)\right),
\end{aligned}
$$

что невозможно. Если подслово $\left(z_{1}\left(z_{2} z_{3}\right)\right) z_{4}$ слова $w\left(\vec{e}, e_{1}\right)$ имеет вид 8$)$, то должно выполняться ложное равенство $x_{u+v+58} x_{u+v+59}=e$. Итак, мы показали, что случаи 1)-8) невозможны. Следовательно, $t_{u}^{v}\left(x_{1}, \ldots, x_{u+v+60}\right)=w_{1}\left(\vec{e}, e_{1}\right)$. База индукции доказана. 
Допустим, что утверждение леммы справедливо для некоторого $n$. Покажем, что оно вьполняется и для $n+1$. В самом деле, пусть $t_{u}^{v}\left(x_{1}, \ldots, x_{u+v+60}\right)$ является подсловом слова $w_{1}\left(\vec{e}, e_{1}\right) \ldots w_{n+1}\left(\vec{e}, e_{1}\right)$. Заметим, что всякое собственное подслово слова $w_{1}\left(\vec{e}, e_{1}\right) \ldots w_{n+1}\left(\vec{e}, e_{1}\right)$ должно быть также и подсловом слова $w_{1}\left(\vec{e}, e_{1}\right) \ldots w_{n}\left(\vec{e}, e_{1}\right)$ или слова $w_{n+1}\left(\vec{e}, e_{1}\right)$. Следовательно, по предположению индукции слово $t_{u}^{v}\left(x_{1}, \ldots\right.$, $\left.x_{u+v+60}\right)$ является собственным подсловом слова $w_{1}\left(\vec{e}, e_{1}\right) \ldots w_{n+1}\left(\vec{e}, e_{1}\right)$ тогда и только тогда, когда существует $i \in\{1, \ldots, n+1\}$ такое, что $t_{u}^{v}\left(x_{1}, \ldots, x_{u+v+60}\right)=w_{i}\left(\vec{e}, e_{1}\right)$. Пусть теперь $t_{u}^{v}\left(x_{1}, \ldots, x_{u+v+60}\right)=w_{1}\left(\vec{e}, e_{1}\right) \ldots w_{n+1}\left(\vec{e}, e_{1}\right)$. Тогда

$$
\begin{gathered}
x_{u+v+60}=w_{n+1}\left(\vec{e}, e_{1}\right), \\
\left(\left(\ldots\left(\left(s_{1}\left(s_{3}\left(s_{1}\left(x_{1}, \ldots, x_{30}\right), x_{31}, \ldots, x_{v+30}\right), x_{v+31}, \ldots, x_{v+60}\right) x_{v+61}\right) x_{v+62}\right)\right.\right. \\
\left.\left.\left.\quad\left(x_{v+63} x_{v+64}\right)\right) x_{v+65} \ldots\right) x_{u+v+55}\right)\left(\left(x_{u+v+56} x_{u+v+57}\right)\left(x_{u+v+58} x_{u+v+59}\right)\right) \\
=w_{1}\left(\vec{e}, e_{1}\right) \ldots w_{n+1}\left(\vec{e}, e_{1}\right) .
\end{gathered}
$$

Пусть $n=1$. Тогда из равенства

$$
\begin{aligned}
& \left(( \ldots ) \left(\left(\left(s_{1}\left(s_{3}\left(s_{1}\left(x_{1}, \ldots, x_{30}\right), x_{31}, \ldots, x_{v+30}\right), x_{v+31}, \ldots, x_{v+60}\right) x_{v+61}\right) x_{v+62}\right)\right.\right. \\
& \left.\left.\left.\quad\left(x_{v+63} x_{v+64}\right)\right) x_{v+65} \ldots\right) x_{u+v+55}\right)\left(\left(x_{u+v+56} x_{u+v+57}\right)\left(x_{u+v+58} x_{u+v+59}\right)\right) \\
& \quad=w_{1}\left(\vec{e}, e_{1}\right) \ldots w_{n+1}\left(\vec{e}, e_{1}\right)
\end{aligned}
$$

получаем $\left(x_{u+v+56} x_{u+v+57}\right)\left(x_{u+v+58} x_{u+v+59}\right)=e$, что невозможно. Допустим, что $n>1$. Тогда из равенства

$$
\begin{aligned}
& \left(( \ldots ) \left(\left(\left(s_{1}\left(s_{3}\left(s_{1}\left(x_{1}, \ldots, x_{30}\right), x_{31}, \ldots, x_{v+30}\right), x_{v+31}, \ldots, x_{v+60}\right) x_{v+61}\right) x_{v+62}\right)\right.\right. \\
& \left.\left.\left.\quad\left(x_{v+63} x_{v+64}\right)\right) x_{v+65} \ldots\right) x_{u+v+55}\right)\left(\left(x_{u+v+56} x_{u+v+57}\right)\left(x_{u+v+58} x_{u+v+59}\right)\right) \\
& \quad=w_{1}\left(\vec{e}, e_{1}\right) \ldots w_{n+1}\left(\vec{e}, e_{1}\right)
\end{aligned}
$$

вытекает, что $\left(x_{u+v+56} x_{u+v+57}\right)\left(x_{u+v+58} x_{u+v+59}\right)=w_{n}\left(\vec{e}, e_{1}\right)$, а значит, должно выполняться ложное равенство $x_{u+v+58} x_{u+v+59}=e$. Поэтому $t_{u}^{v}\left(x_{1}, \ldots, x_{u+v+60}\right)$ не равно $w_{1}\left(\vec{e}, e_{1}\right) \ldots w_{n+1}\left(\vec{e}, e_{1}\right)$. Лемма 3 доказана.

Рассмотрим случай многообразия $\mathfrak{M}^{p+6}$. Многообразия $\mathfrak{B}^{p+6}$ и $\mathfrak{D}^{p+6}$ рассматриваются аналогично. Так как многочлен $h_{1}$ получен из тождества многообразия $\mathfrak{M}^{p+6}$, TO

$$
h_{1}=w_{1}\left(\vec{x}, x_{1}\right) \ldots w_{n}\left(\vec{x}, x_{1}\right)+w_{n+1}\left(\vec{x}, x_{1}\right) \ldots w_{n+k}\left(\vec{x}, x_{1}\right) .
$$

Поэтому в силу леммы 3 одночлен многочлена $h_{1}$ является подсловом слова вида $w_{l_{1}}(\vec{e}$, $\left.e_{1}\right) \ldots w_{l_{p}}\left(\vec{e}, e_{1}\right)$ тогда и только тогда, ког да это $w_{1}\left(\vec{e}, e_{1}\right) \ldots w_{n}\left(\vec{e}, e_{1}\right)$ или $w_{n+1}\left(\vec{e}, e_{1}\right) \ldots$ $w_{n+k}\left(\vec{e}, e_{1}\right)$. Следовательно, если существует $i$ такое, что $x_{i}$ не содержит одночлена $e$, то $f_{1}=0, f_{2}=u_{11} h_{1} u_{12}$. Пусть теперь для любого $i x_{i}=e+x_{i}^{*}$, где $x_{i}^{*}-$ многочлен, не содержащий одночлена $e$. Тогда представим многочлен $h_{1}$ в виде

$$
w_{1}\left(\vec{e}, e_{1}\right) \ldots w_{n}\left(\vec{e}, e_{1}\right)+w_{n+1}\left(\vec{e}, e_{1}\right) \ldots w_{n+k}\left(\vec{e}, e_{1}\right)+f\left(\vec{x}^{*}\right) \text {. }
$$

Легко понять, что $u_{11} f\left(\vec{x}^{*}\right) u_{12}$ - подмногочлен многочлена $f_{2}$. Рассмотрим многочлен $u_{11}\left(w_{1}\left(\vec{e}, e_{1}\right) \ldots w_{n}\left(\vec{e}, e_{1}\right)+w_{n+1}\left(\vec{e}, e_{1}\right) \ldots w_{n+k}\left(\vec{e}, e_{1}\right)\right) u_{12}$. 
ЛЕмма 4. Если для некоторого слова $w_{1}\left(\vec{e}, e_{1}\right) \ldots w_{n}\left(\vec{e}, e_{1}\right)$ при $n>1$ существует слово $w_{l_{1}}\left(\vec{e}, e_{1}\right) \ldots w_{l_{p}}\left(\vec{e}, e_{1}\right)$ такое, что

$$
u_{11}\left(w_{1}\left(\vec{e}, e_{1}\right) \ldots w_{n}\left(\vec{e}, e_{1}\right)\right) u_{12}=w_{l_{1}}\left(\vec{e}, e_{1}\right) \ldots w_{l_{p}}\left(\vec{e}, e_{1}\right)
$$

то для любого слова $w_{a_{1}}\left(\vec{e}, e_{1}\right) \ldots w_{a_{r}}\left(\vec{e}, e_{1}\right)$ найдется слово $w_{b_{1}}\left(\vec{e}, e_{1}\right) \ldots w_{b_{q}}\left(\vec{e}, e_{1}\right)$ такое, что выполняется равенство

$$
w_{b_{1}}\left(\vec{e}, e_{1}\right) \ldots w_{b_{q}}\left(\vec{e}, e_{1}\right)=u_{11}\left(w_{a_{1}}\left(\vec{e}, e_{1}\right) \ldots w_{a_{r}}\left(\vec{e}, e_{1}\right)\right) u_{12}
$$

ДокАЗАТЕЛЬСтво. Из равенства

$$
u_{11}\left(w_{1}\left(\vec{e}, e_{1}\right) \ldots w_{n}\left(\vec{e}, e_{1}\right)\right) u_{12}=w_{l_{1}}\left(\vec{e}, e_{1}\right) \ldots w_{l_{p}}\left(\vec{e}, e_{1}\right)
$$

получаем, что слово $w_{1}\left(\vec{e}, e_{1}\right) \ldots w_{n}\left(\vec{e}, e_{1}\right)$ является собственньм подсловом слова $w_{l_{1}}(\vec{e}$, $\left.e_{1}\right) \ldots w_{l_{p}}\left(\vec{e}, e_{1}\right)$. Легко понять, что произвольное собственное подслово слова $w_{l_{1}}\left(\vec{e}, e_{1}\right)$ $\ldots w_{l_{p}}\left(\vec{e}, e_{1}\right)$ либо является подсловом слова $w_{l_{j}}\left(\vec{e}, e_{1}\right)$ для некоторого $j \in\{1, \ldots, p\}$, либо имеет вид $w_{l_{1}}\left(\vec{e}, e_{1}\right) \ldots w_{l_{p_{0}}}\left(\vec{e}, e_{1}\right)$, где $p_{0}<p$. Из леммы 3 очевидным образом вытекает, что $w_{1}\left(\vec{e}, e_{1}\right) \ldots w_{n}\left(\vec{e}, e_{1}\right)$ не является подсловом слова $w_{l_{j}}\left(\vec{e}, e_{1}\right)$. Следовательно,

$$
w_{1}\left(\vec{e}, e_{1}\right) \ldots w_{n}\left(\vec{e}, e_{1}\right)=w_{l_{1}}\left(\vec{e}, e_{1}\right) \ldots w_{l_{p_{0}}}\left(\vec{e}, e_{1}\right),
$$

а значит, для любого слова $w_{a_{1}}\left(\vec{e}, e_{1}\right) \ldots w_{a_{r}}\left(\vec{e}, e_{1}\right)$ имеем

$$
u_{11}\left(w_{a_{1}}\left(\vec{e}, e_{1}\right) \ldots w_{a_{r}}\left(\vec{e}, e_{1}\right)\right) u_{12}=w_{a_{1}}\left(\vec{e}, e_{1}\right) \ldots w_{a_{r}}\left(\vec{e}, e_{1}\right) w_{l_{p_{0}+1}}\left(\vec{e}, e_{1}\right) \ldots w_{l_{p}}\left(\vec{e}, e_{1}\right) .
$$

Лемма 4 доказана.

Предположим, что слово $u_{11}\left(w_{1}\left(\vec{e}, e_{1}\right) \ldots w_{n}\left(\vec{e}, e_{1}\right)\right) u_{12}$ представимо в виде $w_{l_{1}}\left(\vec{e}, e_{1}\right)$ $\ldots w_{l_{0}}\left(\vec{e}, e_{1}\right)$. Тогда и слово $u_{11}\left(w_{n+1}\left(\vec{e}, e_{1}\right) \ldots w_{n+k}\left(\vec{e}, e_{1}\right)\right) u_{12}$ в силу леммы 4 представимо в аналогичном виде. При этом, как нетрудно убедиться,

$$
\begin{aligned}
& u_{11}\left(w_{1}\left(\vec{e}, e_{1}\right) \ldots w_{n}\left(\vec{e}, e_{1}\right)\right) u_{12} \\
& =w_{1}\left(\vec{e}, e_{1}\right) \ldots w_{n}\left(\vec{e}, e_{1}\right) w_{l_{p_{0}+1}}\left(\vec{e}, e_{1}\right) \ldots w_{l_{p}}\left(\vec{e}, e_{1}\right), \\
& u_{11}\left(w_{n+1}\left(\vec{e}, e_{1}\right) \ldots w_{n+k}\left(\vec{e}, e_{1}\right)\right) u_{12} \\
& =w_{n+1}\left(\vec{e}, e_{1}\right) \ldots w_{n+k}\left(\vec{e}, e_{1}\right) w_{l_{p_{0}+1}}\left(\vec{e}, e_{1}\right) \ldots w_{l_{p}}\left(\vec{e}, e_{1}\right) \text {. }
\end{aligned}
$$

Допустим, что одночлен $w_{1}\left(\vec{e}, e_{1}\right) \ldots w_{n}\left(\vec{e}, e_{1}\right) w_{l_{p_{0}+1}}\left(\vec{e}, e_{1}\right) \ldots w_{l_{p}}\left(\vec{e}, e_{1}\right)$ может быть получен конечньм числом перестановок вида

$$
\begin{aligned}
& A\left(\vec{e}, e_{1}\right) B\left(\vec{e}, e_{1}\right) \leftrightarrow B\left(\vec{e}, e_{1}\right) A\left(\vec{e}, e_{1}\right), \quad C\left(\vec{e}, e_{1}\right) D\left(\vec{e}, e_{1}\right) \leftrightarrow D\left(\vec{e}, e_{1}\right) C\left(\vec{e}, e_{1}\right), \\
& A\left(\vec{e}, e_{1}\right) D\left(\vec{e}, e_{1}\right) \leftrightarrow D\left(\vec{e}, e_{1}\right) A\left(\vec{e}, e_{1}\right), \quad B\left(\vec{e}, e_{1}\right) C\left(\vec{e}, e_{1}\right) \leftrightarrow C\left(\vec{e}, e_{1}\right) B\left(\vec{e}, e_{1}\right)
\end{aligned}
$$

из одночлена вида $Q_{l}\left(\vec{e}, e_{1}\right) A^{r_{1}}\left(\vec{e}, e_{1}\right) B^{r_{2}}\left(\vec{e}, e_{1}\right) C\left(\vec{e}, e_{1}\right) D\left(\vec{e}, e_{1}\right)$. Легко убедиться, что тогда и одночлен $w_{n+1}\left(\vec{e}, e_{1}\right) \ldots w_{n+k}\left(\vec{e}, e_{1}\right) w_{l_{p_{0}+1}}\left(\vec{e}, e_{1}\right) \ldots w_{l_{p}}\left(\vec{e}, e_{1}\right)$ может быть получен аналогичньм образом. Следовательно, либо $f_{1}=0, f_{2}=u_{11} h_{1} u_{12}$, либо $f_{1}=$ $u_{11}\left(w_{1}\left(\vec{e}, e_{1}\right) \ldots w_{n}\left(\vec{e}, e_{1}\right)\right) u_{12}+u_{11}\left(w_{n+1}\left(\vec{e}, e_{1}\right) \ldots w_{n+k}\left(\vec{e}, e_{1}\right)\right) u_{12}, f_{2}=u_{11}\left(f\left(\vec{x}^{*}\right)\right) u_{12}$. 
Легко понять, что из равенства

$$
\begin{aligned}
& Q_{i}\left(\vec{e}, e_{1}\right) A^{\xi_{1}}\left(\vec{e}, e_{1}\right) B^{\xi_{2}}\left(\vec{e}, e_{1}\right) C\left(\vec{e}, e_{1}\right) D\left(\vec{e}, e_{1}\right) \\
& \quad+Q_{j}\left(\vec{e}, e_{1}\right) A^{\eta_{1}}\left(\vec{e}, e_{1}\right) B^{\eta_{2}}\left(\vec{e}, e_{1}\right) C\left(\vec{e}, e_{1}\right) D\left(\vec{e}, e_{1}\right)=f_{1}+f_{2}
\end{aligned}
$$

вытекает $f_{2}=0$. Пусть $h-$ многочлен, полученньй из многочлена

$$
\begin{aligned}
& Q_{i}\left(\vec{e}, e_{1}\right) A^{\xi_{1}}\left(\vec{e}, e_{1}\right) B^{\xi_{2}}\left(\vec{e}, e_{1}\right) C\left(\vec{e}, e_{1}\right) D\left(\vec{e}, e_{1}\right) \\
& \quad+Q_{j}\left(\vec{e}, e_{1}\right) A^{\eta_{1}}\left(\vec{e}, e_{1}\right) B^{\eta_{2}}\left(\vec{e}, e_{1}\right) C\left(\vec{e}, e_{1}\right) D\left(\vec{e}, e_{1}\right)
\end{aligned}
$$

при помоши перестановок вида

$$
\begin{array}{ll}
A\left(\vec{e}, e_{1}\right) B\left(\vec{e}, e_{1}\right) \leftrightarrow B\left(\vec{e}, e_{1}\right) A\left(\vec{e}, e_{1}\right), & C\left(\vec{e}, e_{1}\right) D\left(\vec{e}, e_{1}\right) \leftrightarrow D\left(\vec{e}, e_{1}\right) C\left(\vec{e}, e_{1}\right), \\
A\left(\vec{e}, e_{1}\right) D\left(\vec{e}, e_{1}\right) \leftrightarrow D\left(\vec{e}, e_{1}\right) A\left(\vec{e}, e_{1}\right), & B\left(\vec{e}, e_{1}\right) C\left(\vec{e}, e_{1}\right) \leftrightarrow C\left(\vec{e}, e_{1}\right) B\left(\vec{e}, e_{1}\right),
\end{array}
$$

a $h^{1}, h^{2}$ - его одночлены. Индукцией по числу $d$ одночленов в многочлене $f_{1}$ покажем, что если вьполняется равенство $h=f_{1}$, то существует конечное выгисление на машине $M$, при помощи которого она переходит из конфигурации $q_{i} \xi_{1} \xi_{2}$ в конфигурацию $q_{j} \eta_{1} \eta_{2}$, или наоборот. Предположим, что $d=0$. Тогда утверждение очевидно. Допустим, что утверждение справедливо для некоторого числа одночленов $d$. Покажем, что оно выполняется и для $d+1$. Равенство $h=f_{1}$ можно представить в виде $h^{1}+h^{2}=$ $\sum_{i, j} v_{i_{1}} h_{j} v_{i_{2}}$. В силу свободы кольца $F \Re_{2}$ существуют $i, j$ такие, что $h^{1}-$ одночлен многочлена $v_{i_{1}} h_{j} v_{i_{2}}$. Без ограничения общности можно считать, что $i=j=1$. В зависимости от того, из тождества какого многообразия получен многочлен $h_{1}$, следует рассмотреть три случая. Допустим, что многочлен $h_{1}$ получен из тождества многообразия $\mathfrak{M}^{p+6}$. Тогда, как замечено выше, многочлен $h_{1}$ является суммой двух одночленов. Обозначим их через $h_{1}^{1}$ и $h_{1}^{2}$. Поскольку $h^{1}$ - одночлен многочлена $v_{1_{1}} h_{1} v_{1_{2}}$, то можно считать, что $h^{1}=v_{1_{1}} h_{1}^{1} v_{1_{2}}$. Поэтому из равенства $h^{1}+h^{2}=\sum_{i, j} v_{i_{1}} h_{j} v_{i_{2}}$ получаем

$$
\begin{aligned}
h^{2}+v_{1_{1}} h_{1}^{2} v_{1_{2}} & =h^{1}+h^{2}+v_{1_{1}} h_{1}^{1} v_{1_{2}}+v_{1_{1}} h_{1}^{2} v_{1_{2}} \\
& =\sum_{i, j} v_{i_{1}} h_{j} v_{i_{2}}+v_{1_{1}} h_{1}^{1} v_{1_{2}}+v_{1_{1}} h_{1}^{2} v_{1_{2}}=\sum_{i>1, j} v_{i_{1}} h_{j} v_{i_{2}} .
\end{aligned}
$$

Покажем, что многочлен $h^{2}+v_{1_{1}} h_{1}^{2} v_{1_{2}}$ может быть получен при помоши указанных вьше перестановок из многочлена

$$
\begin{aligned}
& Q_{a}\left(\vec{e}, e_{1}\right) A^{\alpha_{1}}\left(\vec{e}, e_{1}\right) B^{\alpha_{2}}\left(\vec{e}, e_{1}\right) C\left(\vec{e}, e_{1}\right) D\left(\vec{e}, e_{1}\right) \\
& \quad+Q_{b}\left(\vec{e}, e_{1}\right) A^{\beta_{1}}\left(\vec{e}, e_{1}\right) B^{\beta_{2}}\left(\vec{e}, e_{1}\right) C\left(\vec{e}, e_{1}\right) D\left(\vec{e}, e_{1}\right)
\end{aligned}
$$

для некоторых $a, b, \alpha_{1}, \alpha_{2}, \beta_{1}, \beta_{2}$. Предположим, что одночлен $h^{1}$ получен из одночлена $Q_{i}\left(\vec{e}, e_{1}\right) A^{\xi_{1}}\left(\vec{e}, e_{1}\right) B^{\xi_{2}}\left(\vec{e}, e_{1}\right) C\left(\vec{e}, e_{1}\right) D\left(\vec{e}, e_{1}\right)$. Следует рассмотреть четыре случая в зависимости от конкретного вида тождества многообразия $\mathfrak{M}^{p+6}$. Рассмотрение их аналогично. Поэтому мы ограничимся тождеством

$$
Q_{i}\left(\vec{x}, x_{1}\right) A\left(\vec{x}, x_{1}\right) B\left(\vec{x}, x_{1}\right)=Q_{l}\left(\vec{x}, x_{1}\right) A^{\varepsilon_{1}+1}\left(\vec{x}, x_{1}\right) B^{\varepsilon_{2}+1}\left(\vec{x}, x_{1}\right) .
$$


Так как $h_{1}$ соответствует этому тождеству, то слово $v_{1_{1}} h_{1}^{2} v_{1_{2}}$ может быть получено только из слова $Q_{l}\left(\vec{e}, e_{1}\right) A^{\xi_{1}+\varepsilon_{1}}\left(\vec{x}, x_{1}\right) B^{\xi_{2}+\varepsilon_{2}}\left(\vec{x}, x_{1}\right)$. Следовательно, $a=j, b=l$, $\alpha_{1}=\eta_{1}, \alpha_{2}=\eta_{2}, \beta_{1}=\xi_{1}+\varepsilon_{1}, \beta_{2}=\xi_{2}+\varepsilon_{2}$. Таким образом, к равенству $h^{2}+v_{1_{1}} h_{1}^{2} v_{1_{2}}$ можно применить предположение индукции.

Итак, мы убедились, что при $n \geqslant p$ тождество

$$
\begin{aligned}
& Q_{i}\left(\vec{x}, x_{1}\right) A^{\xi_{1}}\left(\vec{x}, x_{1}\right) B^{\xi_{2}}\left(\vec{x}, x_{1}\right) C\left(\vec{x}, x_{1}\right) D\left(\vec{x}, x_{1}\right) \\
& \quad=Q_{j}\left(\vec{x}, x_{1}\right) A^{\eta_{1}}\left(\vec{x}, x_{1}\right) B^{\eta_{2}}\left(\vec{x}, x_{1}\right) C\left(\vec{x}, x_{1}\right) D\left(\vec{x}, x_{1}\right)
\end{aligned}
$$

выполняется в кольце $F_{n} \mathfrak{X}_{p}$ тогда и только тогда, когда существует конечное вычисление на машине $M$, при помощи которого она переходит из конфигурации $q_{i} \xi_{1} \xi_{2}$ в конфигурацию $q_{j} \eta_{1} \eta_{2}$, или наоборот. Поэтому тождество

$$
Q_{1}\left(\vec{x}, x_{1}\right) A^{2^{k}}\left(\vec{x}, x_{1}\right) C\left(\vec{x}, x_{1}\right) D\left(\vec{x}, x_{1}\right)=Q_{0}\left(\vec{x}, x_{1}\right) A\left(\vec{x}, x_{1}\right) C\left(\vec{x}, x_{1}\right) D\left(\vec{x}, x_{1}\right)
$$

выполняется тогда и только тогда, когда $k \in P$. Теорема 1 доказана.

Теорема 2. Существует многообразие колеи $\mathfrak{X}$ с неразрешимой эквациональной теорией такое, что для любого натурального п проблема равенства в кольие $F_{n} \mathfrak{X}$ ранга $n$, свободном в многообразии $\mathfrak{X}$, разрешима.

ДОКАЗАТЕЛЬСТво. Пусть

$$
X_{r}\left(x_{1}, \ldots, x_{r+4}\right) \rightleftharpoons x_{r+4}\left(\left(\ldots\left(\left(x_{3}\left(x_{1} x_{2}\right)\right) x_{4}\right) \ldots\right) x_{r+3}\right)
$$

$P$ - некоторое рекурсивно перечислимое нерекурсивное множество натуральных чисел. Обозначим через $\mathfrak{X}_{1}$ многообразие колец, заданное тождествами $X_{r}\left(x_{1}, \ldots, x_{r+4}\right)=0$, где $r \in P$. Пусть $\mathfrak{X}_{2}-$ многообразие колец, заданное тождествами

$$
2 x=0, \quad Y_{r}\left(x_{1}, \ldots, x_{r+4}\right)=0 \text { и } Z_{r}\left(x_{1}, \ldots, x_{r+5}\right)=0,
$$

где $r$ - произвольное натуральное число и для некоторых $i, j \in\{4, \ldots, r\}$ слово $Y_{r}\left(x_{1}\right.$, $\left.\ldots, x_{r+4}\right)$ может быть получено из слова $X_{r}\left(x_{1}, \ldots, x_{r+4}\right)$ заменой $x_{i}$ на $x_{j}$, а слово $Z_{r}\left(x_{1}, \ldots, x_{r+5}\right)$ получено из $X_{r}\left(x_{1}, \ldots, x_{r+4}\right)$ заменой $x_{i}$ на $x_{i} x_{r+5}$. Обозначим через $\mathfrak{X}$ многообразие колец $\mathfrak{X}_{1} \cap \mathfrak{X}_{2}$.

Покажем, что эквациональная теория многообразия $\mathfrak{X}$ неразрешима. Для этого достаточно убедиться в том, что многообразие $\mathfrak{X}$ удовлетворяет тождеству $X_{r}\left(x_{1}, \ldots\right.$, $\left.x_{r+4}\right)=0$ тогда и только тогда, когда $r \in P$. Допустим, что $r \in P$. Тогда многообразие $\mathfrak{X}$ удовлетворяет тождеству $X_{r}\left(x_{1}, \ldots, x_{r+4}\right)=0$ по построению. Пусть теперь многообразие $\mathfrak{X}$ удовлетворяет тождеству $X_{r}\left(x_{1}, \ldots, x_{r+4}\right)=0$ и $r \notin P$. Так как многообразие $\mathfrak{X}$ удовлетворяет тождеству $X_{r}\left(x_{1}, \ldots, x_{r+4}\right)=0$, то этому тождеству удовлетворяет и кольцо $F \mathfrak{X}$ счетного ранга, свободное в многообразии $\mathfrak{X}$. Следовательно, в кольце $F \mathfrak{X}$ выполняется соотношение $X_{r}\left(e_{1}, \ldots, e_{r+4}\right)=0$, где $e_{1}, \ldots, e_{r+4}-$ свободные образующие кольца $F \mathfrak{X}$. Обозначим через $\mathfrak{R}$ многообразие всех колец характеристики 2 . Пусть $F \mathfrak{R}$ - кольцо счетного ранга свободное в многообразии $\mathfrak{R}$. 
Обозначим через $S$ множество многочленов $f(\vec{e})$ таких, что $f(\vec{e})=0$ - соотношение в кольце $F \mathfrak{X}$, полученное подстановкой конкретных значений вместо переменных в одно из тождеств, задающих многообразие $\mathfrak{X}$. Пусть $I$ - идеал, порожденньй множеством $S$ в кольце $F \Re$. Тогда очевидно, что

$$
F \mathfrak{X}=X_{r}\left(e_{1}, \ldots, e_{r+4}\right)=0 \Longleftrightarrow F \Re \models X_{r}\left(e_{1}, \ldots, e_{r+4}\right) \in I .
$$

Вхождение многочлена $X_{r}\left(e_{1}, \ldots, e_{r+4}\right)$ в идеал $I$ равносильно в кольце $F \Re$ равенству вида $X_{r}\left(e_{1}, \ldots, e_{r+4}\right)=\sum_{i, j} u_{i_{1}} h_{j} u_{i_{2}}$, где $u_{i_{1}} h_{j} u_{i_{2}}$ - некоторое произведение многочленов, одним из сомножителей в котором является многочлен $h_{j}, h_{j} \in S$. В силу дистрибутивности можно считать, что все сомножители, кроме $h_{j}$, в произведении $u_{i_{1}} h_{j} u_{i_{2}}$ являются одночленами. Так как в кольце $F \Re$ выполняется равенство $X_{r}\left(e_{1}, \ldots, e_{r+4}\right)=$ $\sum_{i, j} u_{i_{1}} h_{j} u_{i_{2}}$, то существуют $i, j$ такие, что слово $X_{r}\left(e_{1}, \ldots, e_{r+4}\right)$ является одночленом многочлена $u_{i_{1}} h_{j} u_{i_{2}}$. Без ограничения общности можно считать, что $i=j=1$. В зависимости от того, из какого тождества получен многочлен $h_{1}$, следует рассмотреть три случая. Допустим, что многочлен $h_{1}$ получен из тождества вида $X_{p}\left(x_{1}, \ldots, x_{p+4}\right)$ $=0$. Тогда слово $X_{r}\left(e_{1}, \ldots, e_{r+4}\right)$ должно содержать подслово вида $X_{p}\left(x_{1}, \ldots, x_{p+4}\right)$. Предположим теперь, что $X_{p}\left(x_{1}, \ldots, x_{p+4}\right)$ является собственным подсловом слова $X_{r}\left(e_{1}, \ldots, e_{r+4}\right)$. Тогда должно вьполняться одно из следующих равенств:
1) $X_{p}\left(x_{1}, \ldots, x_{p+4}\right)=\left(\ldots\left(\left(e_{3}\left(e_{1} e_{2}\right)\right) e_{4}\right) \ldots\right) e_{k+3}$, где $k \leqslant r$
2) $X_{p}\left(x_{1}, \ldots, x_{p+4}\right)=e_{3}\left(e_{1} e_{2}\right)$;
3) $X_{p}\left(x_{1}, \ldots, x_{p+4}\right)=e_{1} e_{2}$
4) $X_{p}\left(x_{1}, \ldots, x_{p+4}\right)=e_{i}$, где $i \in\{1, \ldots, r+4\}$.

Заметим, что длина слова $X_{p}\left(x_{1}, \ldots, x_{p+4}\right)$ не меньше 4. Следовательно, равенства 2)-4) невозможны. Допустим, что имеет место равенство 1). Тогда должны выполняться следуюшие два равенства:

$$
x_{r+4}=\left(\ldots\left(\left(e_{3}\left(e_{1} e_{2}\right)\right) e_{4}\right) \ldots\right) e_{k+2}, \quad\left(\ldots\left(\left(x_{3}\left(x_{1} x_{2}\right)\right) x_{4}\right) \ldots\right) x_{r+3}=e_{k+3} .
$$

Заметим, что длина $\left(\ldots\left(\left(x_{3}\left(x_{1} x_{2}\right)\right) x_{4}\right) \ldots\right) x_{r+3}$ не меньше 3 . Следовательно, равенство $\left(\ldots\left(\left(x_{3}\left(x_{1} x_{2}\right)\right) x_{4}\right) \ldots\right) x_{r+3}=e_{k+3}$ ложно. Полученное противоречие доказьвает, что слово $X_{p}\left(x_{1}, \ldots, x_{p+4}\right)$ не является собственным подсловом слова $X_{r}\left(e_{1}, \ldots, e_{r+4}\right)$. Предположим теперь, что выполняется равенство $X_{p}\left(x_{1}, \ldots, x_{p+4}\right)=X_{r}\left(e_{1}, \ldots, e_{r+4}\right)$. По предположению $p \neq r$. Допустим, что $p>r$. Тогда равенство $X_{p}\left(x_{1}, \ldots, x_{p+4}\right)=$ $X_{r}\left(e_{1}, \ldots, e_{r+4}\right)$ очевидно ложно. Пусть теперь $p<r$. Тогда, как нетрудно убедиться, должны выполняться следуюшие равенства:

1) $x_{p+4-i}=e_{r+4-i}$, где $i \in\{0,1, \ldots, p\}$;

2) $x_{3}\left(x_{1} x_{2}\right)=\left(\ldots\left(\left(e_{3}\left(e_{1} e_{2}\right)\right) e_{4}\right) \ldots\right) e_{r+3-p}$.

Из равенства $x_{3}\left(x_{1} x_{2}\right)=\left(\ldots\left(\left(e_{3}\left(e_{1} e_{2}\right)\right) e_{4}\right) \ldots\right) e_{r+3-p}$ очевидным образом вытекает равенство $x_{1} x_{2}=e_{r+3-p}$, что невозможно. Полученное противоречие доказывает, что слово $X_{p}\left(x_{1}, \ldots, x_{p+4}\right)$ не является подсловом слова $X_{r}\left(e_{1}, \ldots, e_{r+4}\right)$ и, следовательно, слово $X_{r}\left(e_{1}, \ldots, e_{r+4}\right)$ не является одночленом многочлена $h_{1}$, полученного из тождества вида $X_{p}\left(x_{1}, \ldots, x_{p+4}\right)=0$. Случай, когда многочлен $h_{1}$ получен из тождества вида $Y_{p}\left(x_{1}, \ldots, x_{p+4}\right)=0$ или $Z_{p}\left(x_{1}, \ldots, x_{p+5}\right)=0$, рассматривается совершенно аналогично. Поэтому мы его опустим. Итак, мы убедились в том, что слово $X_{r}\left(e_{1}, \ldots, e_{r+4}\right)$ 
не является одночленом многочлена $\sum_{i, j} u_{i_{1}} h_{j} u_{i_{2}}$. Отсюда в силу свободы кольца $F \Re$ вытекает, что равенство $X_{r}\left(e_{1}, \ldots, e_{r+4}\right)=\sum_{i, j} u_{i_{1}} h_{j} u_{i_{2}}$ ложно. Следовательно, многообразие $\mathfrak{X}$ удовлетворяет тождеству $X_{r}\left(x_{1}, \ldots, x_{r+4}\right)=0$ тогда и только тогда, когда $r \in P$. Отсюда в силу нерекурсивности множества $P$ получаем, что эквациональная теория многообразия $\mathfrak{X}$ неразрешима.

Для завершения доказательства теоремы 2 нам осталось показать, что для любого натурального $n$ проблема равенства в кольце $F_{n} \mathfrak{X}$ ранга $n$ свободном в многообразии $\mathfrak{X}$ разрешима. Для этого достаточно показать, что существует алгоритм, определяюший по произвольному многочлену $f(\vec{e})$ вьполняется в кольце $F_{n} \mathfrak{X}$ равенство $f(\vec{e})=0$ или нет. В силу разрешимости проблемы равенства в кольце $F \Re($ см. [2]) существует алгоритм, определяющий по произвольному одночлену многочлена $f(\vec{e})$, содержит он подслово вида $X_{r}\left(x_{1}, \ldots, x_{r+4}\right)$ или нет. Допустим, что некоторьй одночлен многочлена $f(\vec{e})$ не содержит подслова вида $X_{r}\left(x_{1}, \ldots, x_{r+4}\right)$. Тогда очевидно, что $f(\vec{e}) \neq 0$. Пусть теперь произвольный одночлен многочлена $f(\vec{e})$ содержит подслова вида $X_{r}\left(x_{1}, \ldots, x_{r+4}\right)$. Тогда, как нетрудно убедиться, равенство $f(\vec{e})=0$ выполняется тогда и только тогда, когда в каждом одночлене многочлена $f(\vec{e})$ подслово $X_{r}\left(x_{1}, \ldots, x_{r+4}\right)$ имеет вид $Y_{r}\left(x_{1}, \ldots, x_{r+4}\right)$ или $Z_{r}\left(x_{1}, \ldots, x_{r+5}\right)$, или $r+4 \geqslant n$, или $r \in P \cap\{1, \ldots, n-5\}$. Каждое из этих четырех условий алгоритмически распознаваемо. Следовательно, проблема равенства в кольце $F_{n} \mathfrak{X}$ действительно разрешима. Теорема 2 доказана.

\section{СПИСОК ЦИТИРОВАННОЙ ЛИТЕРАТУРЫ}

[1] Попов В. Ю. Эквациональные теории многообразий метабелевых и коммутативных колец // Алгебра и логика. 1995. Т. 34. № 3. С. 347-361.

[2] ЖКуков А.И. Приведенные системы определяющих соотношений в неассоциативных алгебрах // Матем. сб. 1950. Т. 27. № 2. С. 267-280.

Уральский государственный технический университет

Поступило 16.12.1997

Исправленный вариант 26.04.1999 